\title{
Aplicación del modelo 4Q para seleccionar aspirantes a Ingeniería de Sistemas a partir del perfil profesional y ocupacional
}

\section{Selection model for beginners to Systems Engineering using 4Q Model and based on professional and working profile}

\author{
O.I. Trejos, L.E. Muñoz, G.R. Solarte.
}

Recibido: junio 18 de 2020 - Aceptado: junio 28 de 2021

\begin{abstract}
Resumen-Una de las dificultades que se presentan en los programas universitarios es la articulación entre todo el proceso de formación durante el período de formación y el logro del perfil profesional y laboral de los futuros egresados. Este pareciera ser un detalle insignificante pero la ausencia de esta minucia ha llevado a que se tengan que implementar mecanismos de validación y acreditación que posibiliten verificar que lo que se le plantea a un estudiante desde el 1er semestre es lo que va a lograr cuando culmine su plan de estudios y se gradúe. En el presente estudio se han tomado como prueba tres universidades públicas y tres privadas $y$, a partir de sus respectivos perfiles profesionales $y$ laborales, se ha aplicado el modelo $4 Q$ de preferencias de pensamiento bajo una propuesta metodológica con el ánimo de realizar una selección de aspirantes que, en algún sentido, brinden una garantía mayor en el logro de dichos perfiles de manera que los esfuerzos que realice la institución sean, en su mayoría, efectivos. Este planteamiento metodológico parte de la premisa de que, si la selección de aspirantes a un programa de formación profesional tiene unos fundamentos apropiados, el
\end{abstract}

${ }^{1}$ Producto derivado del proyecto de investigación "Desarrollo de un modelo de enseñanza y aprendizaje que transversalice el conocimiento derivado de las ciencias básicas y la programación de computadores en ingeniería de sistemas basado en brain based learning y pensamiento computacional", apoyado por la Universidad Tecnológica de Pereira.

O. I. Trejos, Universidad Tecnológica de Pereira, Pereira, Colombia, email: omartrejos@utp.edu.co.

L. E. Muñoz, Universidad Tecnológica de Pereira, Pereira, Colombia, email: lemunozg@utp.edu.co.

G. R. Solarte, Universidad Tecnológica de Pereira, Pereira, Colombia, email: solarte@utp.edu.co.

Como citar este artículo: Trejos, O. I., Muñoz, L. E., y Solarte, G. R. Aplicación del modelo $4 \mathrm{Q}$ para seleccionar aspirantes a Ingeniería de Sistemas a partir del perfil profesional y ocupacional, Entre Ciencia e Ingeniería, vol. 15 , no. 29 , pp. 37-45, enero-junio 2021

DOI: https://doi.org/10.31908/19098367.1703.

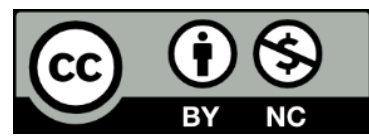

Attribution-NonCommercial 4.0 Intenational (CC By-NC 4.0) proceso podrá llegar a ser tan efectivo que, en gran medida, pueden alcanzarse los perfiles profesionales y laborales que se hayan formulado desde la perspectiva del plan de estudios. La aceptación y efectividad del modelo $4 Q$ en este tipo de procesos, abre las posibilidades de que la metodología propuesta sea útil para las instituciones de educación superior.

Palabras clave -- Aspirantes, ingeniería de sistemas, perfil profesional, plan de estudios, preferencias de pensamiento, programa profesional

Abstract-One of the difficulties that arise in university programs is the articulation between the entire training process during the training period and the achievement of the professional and employment profile of future graduates. This seems to be an insignificant detail, but the absence of this minutia has led to the need to implement validation and accreditation mechanisms that make it possible to verify that what is posed to a student from the 1st semester is what he will achieve when he finishes his curriculum and graduate. In the present study, three public and three private universities have been taken as evidence and, based on their respective professional and labor profiles, the 4Q model of thinking preferences has been applied under a methodological proposal with the aim of making a selection of applicants that, in some sense, provide a greater guarantee in the achievement of such profiles so that the efforts made by the institution are, for the most part, effective. This methodological approach is based on the premise that if the selection of candidates for a vocational training program has appropriate foundations, the process may become so effective that, to a large extent, the professional and labor profiles that have been formulated can be achieved from the perspective of the curriculum. The acceptance and effectiveness of the $4 Q$ model in this type of process open up the possibilities that the proposed methodology will be useful for higher education institutions.

Keywords - Curriculum, systems engineering, professional profile, professional program, thinking preferences, Job profile.

\section{INTRODUCCIÓN}

T A investigación que inspira este artículo tiene por objetivo Lestablecer un mecanismo científico que haga coherente lo que se ofrece al inicio de un programa de formación 
universitario en ingeniería de sistemas, su proceso de formación y el logro de sus perfiles profesionales y laborales tal como lo haya definido el respectivo comité curricular tomando como marco de referencia el modelo $4 \mathrm{Q}$ de preferencias de pensamiento. El presente artículo es un producto derivado de la tesis doctoral "Aprendizaje en Ingeniería: un problema de comunicación" desarrollada por el primer autor del presente artículo [1].

Los programas de ingeniería, en su totalidad, cumplen con los ítems que exigen las normas y los procesos de acreditación nacional e internacional [2], entre los cuales se incluye una vinculación de nuevos estudiantes, un plan de estudios como base para dicha formación y el logro de un perfil profesional y labora. La pregunta de investigación es ¿qué mecanismos tienen los programas de ingeniería de sistemas (que es el caso tipo que nos concita) para que el perfil profesional y laboral sea alcanzado por el proceso de formación imbuido en su plan de estudios y para que se seleccionen a los estudiantes con un perfil académico que encaje plenamente con las expectativas curriculares y de formación de dicho programa de manera que sean más efectivos los esfuerzos que la universidad hace por la excelsitud de su formación?

El objetivo de este estudio es proponer una metodología rigurosa, científica y coherente que permita responder a la pregunta de investigación que se presenta y con ello buscar mayor efectividad a todos los esfuerzos de formación y curriculares que se hacen desde la perspectiva académicoadministrativa de un programa como Ingeniería de Sistemas. La novedad a considerar en este artículo se resume en tres factores: a) no se han realizado estudios sobre la aplicación de un modelo de preferencias de pensamiento (como el modelo 4Q) [3] para realizar la selección de aspirantes a un programa de ingeniería, b) se cumple con los requisitos de los diferentes modelos de acreditación y certificación de los programas asumiendo que el proceso de selección de aspirantes a ingresar al programa, el plan de estudios y todas su actividades asociadas y el perfil profesional y laboral, tienen una línea conductora que no sólo los hace coherentes de cara a los objetivos misionales tanto de la institución como de la profesión sino que también los hace coherentes entre sí, pero ¿en realidad lo son?, c) se propone un modelo para poner a prueba y, con ello, superar las inquietudes que se presentan como novedades en el presente artículo.

La investigación se ha justificado pues, a raíz de un trabajo investigativo basado en el desarrollo de una tesis doctoral, se encontró que algunos de los ítems que contienen todo el corpus de conformación de los programas universitarios no pasan de ser texto escrito y que su cumplimiento, por depender del paso del tiempo, no siempre tiene mecanismos paramétricos que permitan constatarlo [4].

Para desarrollar este artículo se ha acudido a literatura especializada tanto en lo que compete a modelos de aprendizaje [5], al conocimiento y apropiación del modelo 4Q de preferencias de pensamiento y a la teoría de la estructuración curricular en programas universitarios. Es de anotar que se ha escogido el modelo $4 \mathrm{Q}$ de preferencias de pensamiento por su naturaleza científica y por su alta aceptación en el mundo académico e investigativo, además por la confiabilidad en sus resultados a la luz del concepto de calidad educativa [6].
La investigación llega hasta el planteamiento del modelo y la presentación de criterios que garantizan su confiabilidad y cientificidad. Su puesta en escena es todavía motivo de estudio dado que va de la mano del tiempo que transcurre desde cuando los aspirantes ingresan al programa (con todas las variantes que se presentan durante su permanencia en la universidad) hasta cuando obtienen su título profesional y se ubican en la sociedad como nuevos egresados.

La investigación se ha realizado tomando como base universidades públicas y universidades privadas en las cuales, a partir de la definición de su perfil profesional y laboral y de su interpretación desde la perspectiva que brinda el modelo $4 \mathrm{Q}$ de preferencias de pensamiento, se ha planteado un mecanismo para garantizar que dichos perfiles se logren sobre una base científica de análisis de su plan de estudios y adoptando mecanismos de selección de aspirantes que hagan efectivo todo este proceso. Vale la pena anotar que la información sobre la cual se ha desarrollado el modelo que se plantea en el presente artículo proviene de los sitios web institucionales considerándolos como la voz virtual oficial de los programas en mención. De este ejercicio se presentan, como ejemplo, dos universidades en el presente artículo.

\section{MARCO TEÓRICO}

El diseño de un plan de estudios que soporte los objetivos de formación profesional obedece, en la realidad, tanto a metodologías pensadas para tal fin desde la perspectiva de la investigación educativa como a unión de intereses académicos, tecnológicos y personales que finalmente le confieren el corpus que el plan de estudios requiere [7]. Es claro que todo programa de formación tendrá asociado un perfil tanto profesional como laboral que será la impronta con la cual los egresados saldrán a ejercer y a articularse con esa sociedad que los requiere y que, en temas de ingeniería, está ávida de soluciones a los diferentes problemas que poco a poco van emergiendo y aquellos que, con el paso del tiempo, aún no han sido resueltos.

En la mayoría de los programas, tanto nacientes como existentes, se acude a la asesoría de profesionales expertos en el ámbito del diseño curricular para hacer coherente el plan de estudios con los planteamientos que, en términos generales, se quieren alcanzar. Sin embargo, una de las grandes dificultades que se encuentran en los programas de Ingeniería es que quienes saben de Ingeniería no saben de diseño curricular y viceversa, lo cual hace un poco más compleja la situación. A esto se le suma la desconexión existente entre el perfil profesional y laboral, que debe alcanzarse al concluir exitosamente el plan de estudios [8], con la selección de aspirantes a ingresar a un determinado programa de ingeniería, para lo cual deberán clasificarse a los estudiantes más por sus aptitudes que por sus actitudes.

En la metodología que inspira el presente artículo se ha escogido el modelo 4Q de preferencias de pensamiento [9] como la base para clasificar a los estudiantes que aspiran a ingresar a un programa de ingeniería y, a partir del enfoque que provee este modelo, se realizan los análisis pertinentes para que se establezcan criterios que permitan proponer un plan de estudios que, con esta misma perspectiva, permita 
alcanzar tanto el perfil profesional como el perfil laboral, como se explicará más adelante.

En su más simple concepción, el modelo 4Q de preferencias de pensamiento [10] debe su nombre a la formulación de un cerebro que se divide en 4 cuadrantes (4 Quadrants) y que se han denotado con letras [11]: cuadrante A que corresponde al cuadrante lógico, cuadrante $\mathrm{B}$ que corresponde al cuadrante secuencial, cuadrante $\mathrm{C}$ que corresponde al cuadrante social y cuadrante $\mathrm{D}$ que corresponde al cuadrante imaginativo. Estos son los cuatro enfoques que establece el modelo en cuanto a la relación del individuo con su entorno, su forma de concebir dicho entorno y la manera como lo aborda en sus elementos constitutivos. El modelo también propone que, aunque todos los cerebros están conformados por los cuatro cuadrantes, solo uno es el dominante y por ello se llama modelo de preferencias de pensamiento pues este cuadrante dominante es el que "gobierna" sobre los demás y es el que determina esa relación del individuo con su entorno.

Las personas con cuadrante lógico dominante (cuadrante A) son aquellas que siempre quieren conocer muy bien las reglas que existen de por medio, entender de dónde provienen y saber tanto la génesis como los efectos de dichas reglas. Son personas que actúan cuando su lógica ha actuado de primera y que toman la iniciativa cuando los principios, las reglas y las normas que se involucren estén plenamente claras y se sepa el porqué de su existencia. Son personas excelentes planeando, organizando, estructurando y formulando normas. También son muy hábiles entendiendo cómo funcionan las organizaciones, interviniéndolas, mejorándolas, optimizándolas y logrando que sus partes sean un todo estructural en el momento en que se necesite. Tienen un perfil que facilita que asuman el rol de jefes en las organizaciones. Siempre buscan resolver el POR QUÉ de las cosas. Conciben el mundo a partir de sus normas.

Las personas con cuadrante secuencial dominante (cuadrante B) son aquellas que tienen la capacidad de cumplir las órdenes o las instrucciones sin cuestionarlas. No están muy preocupados por la génesis de las normas sino por el cumplimiento de las mismas. Tienen gran capacidad para hacer efectivo los diseños de organización que otros hagan por ella. Acatan lo que se les ordena y siempre buscan que las instrucciones a seguir tengan un sentido de secuencialidad, allende las razones que las inspiren. Conocen bien las normas, las reglas, los estándares y las hacen cumplir. Son excelentes subalternos, aunque no necesariamente llegan a ser buenos jefes. Saben convertirse en la mano derecha y son expertos en la realización de tareas operativas $\mathrm{y}$, mayoritariamente, repetitivas. Poco cuestionan, ellos solo cumplen con el QUÉ. Conciben el mundo a partir de la coherencia de sus normas.

Las personas con cuadrante social dominante (cuadrante C) son aquellas que tienen la capacidad de ser líderes naturales pues saben ganarse la confianza de los demás colegas, compañeros, copartidarios y seres humanos con quienes comparten sus espacios vitales, laborales y profesionales. Tienen la forma de llegar a cada uno hablando en sus propios términos y saben entender a los demás con una pluma que resulta ser de gran utilidad dentro de las organizaciones empresariales. Conocen las normas y saben ajustarlas dependiendo de la conveniencia de las situaciones. Aunque no cumplen literalmente cada regla que se les encomienda, saben cuándo hacer las excepciones para bien de cada persona y para bien de las empresas. Saben encantar a los demás y motivarlos para que hagan las cosas que se requieren. Con frecuencia se ganan el puesto de ser líderes dentro de las empresas. Normalmente acuden al PARA QUÉ y de esta forma saben articular, de manera natural, los intereses de las personas con los intereses de las organizaciones. Conciben el mundo a partir de la interacción entre los seres humanos.

Las personas con cuadrante imaginativo dominante (cuadrante D) son aquellas que siempre están buscando otros horizontes para plantear soluciones y situaciones innovadoras de forma que conciben un mundo cambiante en donde a cada paso se abre una posibilidad que puede optimizarlo y hacer que sea mejor. Se alejan con gran frecuencia de las normas establecidas en su ánimo de pensar en nuevas y mejores cosas para cada persona. Son soñadores y consideran que siempre un mundo mejor es posible. Creen en el ser humano $\mathrm{y}$, por lo tanto, su instinto humanista les hace olvidar, por momentos, los intereses empresariales para sobreponerles los intereses que favorezca a las personas. No siempre es fácil entenderlos pues en muchas situaciones, proponen soluciones inverosímiles que, a la postre, pueden llegar a ser óptimas y mejores que aquellas ideas que ya están funcionando. Ven el mundo con un color diferente al de los demás y por ello las tareas repetitivas, el diseño de normas y el cumplimiento de las mismas no es completamente lo suyo. La interacción social puede llegar a ser uno de sus fuertes siempre que se les pueda entender en la dimensión de su imaginación.

Por su parte, puede decirse que el perfil profesional es la definición clara del conjunto de competencias y habilidades con las cuales cuenta una persona formada en una institución de educación superior para atender las necesidades y problemas que tiene una sociedad tanto en lo que se refiere a lo existente como lo nuevo que va apareciendo [12]. El perfil laboral es el conjunto de tareas específicas (sean éstas repetitivas o no) que una persona, con cierto grado de formación, puede realizar de manera confiable, ética y responsable y sobre las cuales se puede depositar toda la confianza institucional [13].

En cuando a la selección de aspirantes a un programa de Ingeniería algunas universidades han implementado exámenes de conocimientos generales a partir de los cuales se establece una escala cuantitativa que permita el acceso de dichos aspirantes al programa. Lo que se propone es que el modelo $4 \mathrm{Q}$ sirva como referente para un análisis cualitativo tal que el ingreso a la carrera no se haga a partir de la resolución de un conjunto de preguntas sino a partir de una aproximación a la manera como el aspirante concibe el mundo y su coincidencia con lo que se espera alcanzar como perfil profesional y laboral.

Un plan de estudios de puede definir como el conjunto de actividades, asignaturas, conocimientos, metodologías, estrategias y actividades que propone un corpus de áreas obligatorias y básicas y de áreas electivas que forman parte de un currículo con el cual se esperan lograr determinados objetivos de aprendizaje en algún nivel formal de preparación académica [14]. En el plan de estudios se espera que se definan claramente las asignaturas con sus contenidos; la distribución del tiempo y las secuencias que involucra el 
proceso educativo; los logros, las competencias y los conocimientos que los estudiante involucrados deben alcanzar para adquirir el aval de continuar con el siguiente nivel de dicho plan; la metodología que debe aplicarse a cada una de las áreas en donde ha de incluirse el material de apoyo, los libros de referencias y todas las ayudas física y virtuales que se requieran para cumplir con los objetivos definidos [15].

El modelo que se plantea establece como primer paso de ingreso a un programa de ingeniería la aplicación del modelo 4Q como mecanismo de selección. Con esto el aspirante adquiere su status de estudiante y se gana el derecho de recorrer el camino del plan de estudios del Programa de Ingeniería que ha sido diseñado curricularmente para alcanzar un perfil profesional y un perfil laboral con el cual el egresado se podrá articular con las necesidades de la sociedad y podrá ser formulador de soluciones de éstas. El proceso de transformación de aspirante a egresado bajo la óptica del modelo 4Q en su selección y con la coherencia de un plan de estudios que brinde un nivel de garantía aceptable en relación con el cumplimiento del perfil profesional y el perfil laboral es lo que se propone como metodología en este artículo.

\section{Metodología}

Lo primero que debe aclararse es que este análisis se realiza de atrás hacia adelante que, visto desde una perspectiva práctica y algorítmica, podría resumirse en los siguientes pasos:

- Planteamiento del perfil profesional y perfil laboral que se quiere alcanzar

- Desglose de ambos perfiles desde la perspectiva del modelo $4 \mathrm{Q}$, es decir, tomando como base la estructuración del cerebro en los cuatro cuadrantes y las diferentes miradas al mundo que desde cada cuadrante se pueden tener

- Revisión y calificación cuantitativa del desglose logrado tanto en lo absoluto como en su proporcionalidad

- Verificación del cuadrante (o cuadrantes) que hayan obtenido la mayor puntuación

- Revisión del plan de estudios desde la perspectiva de los resultados parciales obtenidos en el ítem anterior

- Recomendación acerca de la estructuración del plan de estudios teniendo en cuenta la recomendación que se deriva de la aplicación el modelo 4Q

- Revisión de los requerimientos de ingreso a la carrera que deben cumplir los aspirantes

- Designación de una metodología científica para perfilar a los estudiantes que aspiran al programa de Ingeniería

Este planteamiento llevaría a pensar en que el esquema planteado muta hacia un redireccionamiento que lo hace más efectivo y más práctico en relación con la articulación entre el perfil del aspirante que quiere ingresar al programa de Ingeniería y los perfiles profesional y laboral que el programa promete alcanzar con la formación que se deriva del desarrollo de todo el plan curricular incluyendo el plan de estudios.

Nótese que el sentido del análisis implica que se conciba en primer lugar al Egresado con una impronta representada en su perfil profesional y perfil laboral y, con esta parte definida, aplicar la metodología se hace muy sencillo puesto que se tiene claramente definido un norte de formación dentro del contexto de un programa de ingeniería. Ahora bien, la concepción del egresado a partir de la definición de su perfil profesional y laboral obedece a la dinámica propia de la institución universitaria, a su historia, a su interacción con el medio, a sus aportes a la sociedad y a la forma como haya liderado procesos académicos, investigativos y de extensión. Variables que se salen del contexto de análisis de esta investigación pero que corresponden a la temática de otro artículo por su importancia en el momento de que se definan las características que identifican y hacen diferente a un programa de educación superior.

El desglose de los perfiles profesional y laboral, que se sugiere en la metodología, se convierte en el gran insumo que transforma la manera de concebir los demás componentes formativos de un programa de formación universitaria puesto que, a partir de éste, se configuran todos los elementos que conforman la estructura curricular incluyendo, como ha de suponerse, el plan de estudios. La revisión y calificación cuantitativa del desglose logrado deberá obedecer a una tarea conjunta realizada preferiblemente por el comité curricular del programa de formación puesto que es la discusión, las diferentes miradas y el sano debate lo que hace que se pueda llegar a una aproximación en lo que se quiere a nivel institucional. Es de anotar que se busca que exista la mayor objetividad posible en esta metodología sin embargo algunos destellos de subjetividad y percepción personal podrían ser ineludibles.

La definición del cuadrante (o cuadrantes) que hayan obtenido la mayor puntuación, luego de este proceso de análisis preferiblemente colectivo, es la piedra angular sobre la cual se construye la proporcionalidad de las líneas de formación en el plan de estudios tomando como referencia la relación porcentual que se observe dicha calificación cuantitativa. La revisión del plan de estudios implicará asumir las conclusiones que, hasta el momento, se hayan alcanzado dado que en todo momento el mediador que sirve de fundamento es el modelo $4 \mathrm{Q}$ de preferencias de pensamiento.

Vale la pena tener en cuenta que la proporcionalidad que se deriva del análisis cuantitativo de los perfiles profesional y laboral a la luz del modelo $4 \mathrm{Q}$ es una sugerencia con un gran fundamento pero que es el comité curricular quien tendrá la autonomía y franquicia de virar los cambios que considere pertinentes hacia el horizonte que crea más oportuno dependiendo del estado de la tecnología, de las realidades sociales, de las condiciones institucionales y de otras variables que no aparecen en este proceso y que, a juicio de ese mismo comité curricular, podrían ser importantes, vinculantes e, incluso, determinantes. Lo que se busca es que se tenga una metodología científicamente fundamentada que posibilite que la relación Aspirante $\leftarrow$ Egresado sea efectiva, coherente y acorde con las políticas académico-administrativas institucionales.

Es de anotar que, sobre la base del análisis inverso que se propone, las pruebas de clasificación de los aspirantes (basados en el modelo 4Q) podrían corresponder tanto a pruebas estandarizadas $4 \mathrm{Q}$ como las que se realizan en la propia metodología de William Herrmann [16] o las pruebas Benziger [17] aunque también podrían diseñarse pruebas 
escritas desarrolladas sobre la base de los perfiles preferentes ideales que se detecten del análisis.

Todo el proceso descrito hasta el momento se hace efectivo cuando se cristaliza en el plan de estudios y cuando se plantean los requerimientos necesarios para ingresar a la carrera en relación con el perfil de los aspirantes y a la luz del modelo 4Q por lo cual es, en este instante, en el cual se debe acoger una metodología para poder perfilar de manera confiable a los alumnos que aspiran ingresar al programa. Se presentan en el numeral anterior un ejemplo práctico realizado con tres universidades en el cual se sugiere todo el proceso. La información respectiva ha sido tomada de los sitios oficiales de las universidades $\mathrm{y}$, por respeto a su privacidad, simplemente se identificarán como las Universidades X, Y y Z.

\section{Resultados}

Se realizarán tres ejemplos completos con información real consultada en los WebSites de tres universidades. La identificación de ellas se oculta por respeto a su privacidad institucional. En la primera universidad (Universidad X) se cuenta con la siguiente información de perfil profesional y ocupacional (que corresponde al perfil laboral) para el programa Ingeniería de Sistemas y Computación.

\section{UNIVERSIDAD X}

Programa Ingeniería de Sistemas y Computación

\section{PERFIL PROFESIONAL}

- Participar en proyectos de investigación en distintas Áreas Tecnológicas.

- Utilizar herramientas computacionales para solucionar problemas de Ingeniería.

- Participar en la creación, diseño y desarrollo de soluciones informáticas.

- Identificar y resolver retos y problemas informáticos y administrativos.

- Participar en la definición de los procedimientos de control interno y auditoría.

- Tener conocimiento y dominio de un idioma extranjero.

- Desarrollar Investigación que genere soluciones en telecomunicaciones, sistemas de información y hardware.

\section{PERFIL OCUPACIONAL}

- El ingeniero de Sistemas y Computación de la U.T.P. estará en capacidad de participar y generar nuevas alternativas en la creación, planeación, análisis, diseño y desarrollo de proyectos informáticos de una organización.

- Enfrentar desafíos que conlleven investigación tecnológica en diversas especialidades de la Ingeniería.

- Aplicar los conocimientos científicos y humanísticos para comprender y dar solución a las diversas situaciones que comprometen el destino de la sociedad.

- Analizar la situación organizacional de acuerdo con su entorno social, político, económico y cultural, determinando las necesidades de información para el buen funcionamiento empresarial.

- Identificar, como buen ciudadano, que profesa el respeto por los demás, las leyes y el civismo.
- Desempeñarse como profesional eficiente y responsable que genere progreso y riqueza tanto en el ámbito empresarial como nacional.

Ahora se va a clasificar cada ítem tanto del perfil profesional como del perfil ocupacional (laboral) por su relación con cada uno de los cuadrantes del modelo 4Q las características científicas planteadas y el análisis con la descripción de cada ítem. La tabla IA presenta la información de manera resumida de la relación perfil profesional y modelo 4Q. Por su parte la tabla IB presenta la respectiva información en relación con el análisis sobre el perfil ocupacional (laboral).

El primer ítem se ha identificado con el cuadrante $\mathrm{C}$ (cuadrante social) debido a que la participación en proyectos de investigación en distintas áreas tecnológicas involucra una componente social alta de forma que el egresado pueda interactuar con otras personas dentro de un marco de buenas convergencias, sano debate y cordiales discrepancias a la luz de su desempeño profesional. Por su parte el uso de herramientas computacionales para resolver problemas de ingeniería implica ajustarse a las posibilidades que dichas herramientas proveen y apropiar plenamente el concepto de la algoritmización de las soluciones de problemas. Ambas situaciones implican una componente fundamentalmente secuencial y por eso se ha asociado con el cuadrante B.

TABLA IA

RELACIÓN PERFIL PROFESIONAL - MODELO 4Q UNIVERSIDAD X.

\begin{tabular}{|c|c|c|c|c|}
\hline \multirow{2}{*}{ Descripción Perfil Profesional } & \multicolumn{4}{|c|}{ Modelo 4Q } \\
\hline & $\mathbf{A}$ & $\mathbf{B}$ & $\mathbf{C}$ & $\mathbf{D}$ \\
\hline $\begin{array}{l}\text { 1. Participar en proyectos de investigación en } \\
\text { distintas Áreas Tecnológicas. }\end{array}$ & & & $\mathrm{X}$ & \\
\hline $\begin{array}{l}\text { 2. Usar de herramientas computacionales para } \\
\text { resolver problemas de Ingeniería. }\end{array}$ & & $\mathrm{X}$ & & \\
\hline $\begin{array}{l}\text { 3. Participar en la creación, diseño y desarrollo } \\
\text { de soluciones informáticas. }\end{array}$ & & & & $\mathrm{X}$ \\
\hline $\begin{array}{l}\text { 4. Identificar y resolver retos y problemas } \\
\text { informáticos y administrativos. }\end{array}$ & $\mathrm{X}$ & & & \\
\hline $\begin{array}{l}\text { 5. Participar en la definición de los } \\
\text { procedimientos de control interno y } \\
\text { auditoría. }\end{array}$ & & $X$ & & \\
\hline $\begin{array}{l}\text { 6. Tener conocimiento y dominio de un idioma } \\
\text { extranjero. }\end{array}$ & & & $\mathrm{X}$ & \\
\hline $\begin{array}{l}\text { 7. Desarrollar Investigación que genere } \\
\text { soluciones en telecomunicaciones, } \\
\text { sistemas de información y hardware. }\end{array}$ & $\mathrm{X}$ & & & \\
\hline
\end{tabular}

Estas dos explicaciones anteriores podrían tener algún debate, que será excluido del desarrollo de este artículo, pues las reflexiones al respecto son las que se derivan de la experiencia y conocimiento del autor del mismo. Se podría pensar en un nivel de detalle mayor para aproximarse más a los perfiles que pueden influir o inspirar cada ítem, sin embargo, algunos ejercicios realizados con excelso nivel de filigrana han llegado a las mismas conclusiones que se presentan en la tabla IA. De manera similar a los dos primeros, ítems se realiza el análisis de los demás ítems que conforman el perfil profesional de la Universidad X.

En el caso del perfil ocupacional, se busca analizar cuál podría ser el cuadrante (o los cuadrantes, pues podrían ser varios) que mayormente inciden en el ítem estudiado. Por ejemplo, el primer ítem involucra la participación y generación de nuevas alternativas en la creación, planeación, análisis, diseño y desarrollo de proyectos informáticos de una 
organización lo cual pareciera concederle mayor relevancia al cuadrante Lógico (cuadrante A) por la naturaleza propia de su definición y por las características científicas que lo identifican.

TABLA IB

RELACIÓN PERFIL OCUPACIONAL - MODELO 4Q - UNIVERSIDAD X

\begin{tabular}{|c|c|c|c|c|}
\hline \multirow{2}{*}{ Descripción Perfil Ocupacional } & \multicolumn{4}{|c|}{ Modelo 4Q } \\
\hline & $\mathbf{A}$ & B & $\mathbf{C}$ & D \\
\hline $\begin{array}{l}\text { 1. Participar y generar nuevas alternativas en la } \\
\text { creación, planeación, análisis, diseño y } \\
\text { desarrollo de proyectos informáticos de una } \\
\text { organización. }\end{array}$ & $X$ & & & \\
\hline $\begin{array}{l}\text { 2. Enfrentar desafíos que conlleven investigación } \\
\text { tecnológica en diversas especialidades de la } \\
\text { Ingeniería. }\end{array}$ & & & & X \\
\hline $\begin{array}{l}\text { 3. Aplicar los conocimientos científicos y } \\
\text { humanísticos para comprender y dar solución } \\
\text { a las diversas situaciones que comprometen el } \\
\text { destino de la sociedad. }\end{array}$ & & $X$ & & \\
\hline $\begin{array}{l}\text { 4. Analizar la situación organizacional de acuerdo } \\
\text { a su entorno social, político, económico y } \\
\text { cultural, determinando las necesidades de } \\
\text { información para el buen funcionamiento } \\
\text { empresarial. }\end{array}$ & $X$ & & & \\
\hline $\begin{array}{l}\text { 5. Identificar, como buen ciudadano, que profesa } \\
\text { el respeto por los demás, las leyes y el } \\
\text { civismo. }\end{array}$ & & $X$ & & \\
\hline $\begin{array}{l}\text { 6. Desempeñarse como profesional eficiente y } \\
\text { responsable que genere progreso y riqueza } \\
\text { tanto en el ámbito empresarial como nacional. }\end{array}$ & & & $X$ & \\
\hline
\end{tabular}

De la misma manera, si se toma el segundo ítem que indica "Enfrentar desafíos que conlleven investigación tecnológica en diversas especialidades de la Ingeniería", la experiencia dice que se requiere una gran componente imaginativa, en lo fundamental, para cumplir con esa característica del perfil sin desconocer que otros elementos son heredados de los otros cuadrantes pues en lo lógico deberán conocerse bien la génesis de las reglas que implican la investigación tecnológica, en lo secuencial deberá tenerse un gran conocimiento de los pasos a realizar al momento de iniciar una investigación científica y en lo social deberá tenerse la capacidad de seducir a quienes se vean afectados por los resultados y puesta en marcha de dicha investigación, todo indica que es el cuadrante imaginativo (cuadrante D) el que tendrá la firme tarea de coordinar los otros tres cuadrantes para bien de las soluciones que se deben implementar como resultado de la investigación tecnológica.

La tabla IC presenta los resultados resumidos del análisis realizado a los perfiles profesional y ocupacional (laboral) que se han tomado como ejemplo.

Como puede observarse en la tabla $1 \mathrm{C}$, la componente mayor de los perfiles profesional y ocupacional corresponde a propósitos que parecieran enmarcarse, a la luz de un análisis detenido y fundamentado en el modelo 4Q, en la definición y características del cuadrante A (cuadrante Lógico). En su orden ocupan un segundo nivel de inferencia los cuadrantes $\mathrm{B}$ y C (secuencial y social) respectivamente y, de acuerdo con las definiciones planteadas por el comité curricular para el programa Ingeniería de Sistemas y Computación de la Universidad $\mathrm{X}$ el cuadrante que menos se requiere pareciera ser el Imaginativo (cuadrante D).
TABLA IC

\begin{tabular}{|c|c|c|c|c|c|}
\hline Perfil & Ítem & $\begin{array}{c}\text { Cuad } \\
\text { A } \\
\text { Lóg }\end{array}$ & $\begin{array}{c}\text { Cuad } \\
\text { B } \\
\text { Sec }\end{array}$ & $\begin{array}{c}\text { Cuad } \\
\text { C } \\
\text { Soc }\end{array}$ & $\begin{array}{c}\text { Cuad } \\
\text { D } \\
\text { Imag }\end{array}$ \\
\hline \multirow{7}{*}{ PROFESIONAL } & 1 & & & $\mathrm{X}$ & \\
\hline & 2 & & $\mathrm{X}$ & & \\
\hline & 3 & & & & $\mathrm{X}$ \\
\hline & 4 & $X$ & & & \\
\hline & 5 & & $\mathrm{X}$ & & \\
\hline & 6 & & & $\mathrm{X}$ & \\
\hline & 7 & $\mathrm{X}$ & & & \\
\hline \multirow{6}{*}{ OCUPACIONAL } & 1 & $X$ & & & \\
\hline & 2 & & & & $\mathrm{X}$ \\
\hline & 3 & & $\mathrm{X}$ & & \\
\hline & 4 & $\mathrm{X}$ & & & \\
\hline & 5 & & $\mathrm{X}$ & & \\
\hline & 6 & & & $\mathrm{X}$ & \\
\hline \multicolumn{2}{|c|}{ TOT A L E S } & 4 & 3 & 3 & 2 \\
\hline \multicolumn{2}{|c|}{ PORCENTAJES } & $33 \%$ & $25 \%$ & $25 \%$ & $16 \%$ \\
\hline
\end{tabular}

Esto nos lleva a pensar en que, para alcanzar los perfiles profesional y ocupacional definidos, debe construirse un plan de estudios que podría contener asignaturas que en un $33 \%$ tuvieran un componente altamente lógico, en un $25 \%$ tuvieran una alta componente secuencial, en otro $25 \%$ tuvieran un gran componente social y en un $16 \%$ tuvieran un componente imaginativo. Por tratarse de un programa de Ingeniería de Sistemas y Computación, se podría pensar en asignaturas altamente lógicas como Algoritmia, Aritmética Computacional, programación orientada a objetos y Lógica; por su parte asignaturas altamente secuenciales podrían ser Programación Imperativa, programación funcional, programación de dispositivos móviles, programación orientada a la web; en cuanto a asignaturas con un alto perfil social se podría pensar en Emprendimiento, Administración de Empresas, Análisis de problemas contemporáneos, Humanidades y en lo que se refiere a asignaturas con perfil imaginativo podría pensarse en Desarrollo del Pensamiento, Historia del Arte, Evolución de la Tecnología, Nuevas Tecnologías e Ingeniería del Software, por citar tan sólo algunas de ellas.

Ahora bien, organizando, revisando y evaluando un plan de estudios que cumpla con estas características entonces se podría definir los elementos que permiten perfilar como elegibles a los estudiantes que aspiran ingresar al programa Ingeniería de Sistemas y Computación de esta Universidad X. $\mathrm{Se}$ requiere preparar un examen de ingreso que incluya preguntas con los cuatro componentes (lógico, secuencial, social e imaginativo) y serán elegibles, considerados como candidatos óptimos para su ingreso al programa en mención, aquellos estudiantes que obtengan $33 \%$ de resultados favorables en la parte lógica, $25 \%$ en la parte secuencial, $25 \%$ en la parte social y $16 \%$ en la parte imaginativa. Todo esto se realiza y se propone teniendo en cuenta el perfil profesional y ocupacional que tiene definida la universidad $X$ para el programa en mención. También se podría pensar en que el plan de estudios incluya asignaturas cuyos componentes de contenido, estrategias y actividades, correspondan a los porcentajes dados en los ítems relacionados, es decir, en cada cuadrante del modelo 4Q. En cuanto a la selección de estudiantes también se puede acudir a las pruebas estándar de perfil de pensamiento preferente que se refirieron en párrafos 
anteriores, teniendo en cuenta los porcentajes de lo que se quiere lograr con la formación en el programa indicado. Nótese que la presencia de los cuatro cuadrantes es importante pues son complementarios, a menos que el análisis de los perfiles profesional y ocupacional, indique que alguno de ellos no incide de manera absoluta en la construcción del plan de estudios y sus actividades asociadas. Veamos este mismo análisis en otra universidad, que llamaremos Universidad Y, pero en el mismo programa. Al consultar su website se obtiene la siguiente información oficial.

TABLA IIA

RELACION PERFIL PROFESIONAAL - MODELO 4Q

\begin{tabular}{|c|c|c|c|c|}
\hline \multirow[b]{2}{*}{ Descripción Perfil Profesional } & \multicolumn{4}{|c|}{ Modelo 4Q } \\
\hline & $\begin{array}{c}\text { A } \\
\text { Log }\end{array}$ & $\begin{array}{c}\text { B } \\
\text { Sec }\end{array}$ & $\begin{array}{c}\mathrm{C} \\
\text { Soc }\end{array}$ & $\begin{array}{c}\text { D } \\
\text { Ima }\end{array}$ \\
\hline 1. ...es un profesional ético, & $\mathrm{X}$ & & & \\
\hline 2. responsable, & & & $\mathrm{X}$ & \\
\hline 3. crítico & & $\mathrm{X}$ & & \\
\hline 4. y autónomo; & $\mathrm{X}$ & & & \\
\hline $\begin{array}{l}\text { 5. con facilidad para integrar equipos } \\
\text { interdisciplinarios }\end{array}$ & & & $\mathrm{X}$ & \\
\hline 6. y con capacidad para plantear, & & & & $\mathrm{X}$ \\
\hline 7. desarrollar, & & $\mathrm{X}$ & & \\
\hline 8. implantar, & $\mathrm{X}$ & & & \\
\hline 9. gestionar & & & $\mathrm{X}$ & \\
\hline $\begin{array}{l}\text { 10.y evaluar alternativas de solución a } \\
\text { problemas, necesidades } \\
\text { oportunidades }\end{array}$ & $\mathrm{X}$ & & & \\
\hline 11.de la sociedad & & & $\mathrm{X}$ & \\
\hline $\begin{array}{l}\text { 12.y organizaciones de distintos sectores } \\
\text { de la economía relacionadas }\end{array}$ & $\mathrm{X}$ & & & \\
\hline $\begin{array}{c}\text { 13.con procesos y manejo de la } \\
\text { información }\end{array}$ & & $\mathrm{X}$ & & \\
\hline $\begin{array}{l}\text { 14.mediante la aplicación de sus } \\
\text { conocimientos en ciencias básicas }\end{array}$ & $\mathrm{X}$ & & & \\
\hline $\begin{array}{l}\text { 15.y tecnologías de la información y las } \\
\text { comunicaciones. }\end{array}$ & & $\mathrm{X}$ & & \\
\hline
\end{tabular}

TABLA IIB

RELACION PERFIL OCUPACIONAL - MODELO 4Q

\begin{tabular}{|l|c|c|c|c|}
\hline \multirow{2}{*}{ Descripción Perfil Profesional } & \multicolumn{3}{|c|}{ Modelo 4Q } \\
\cline { 2 - 5 } & $\begin{array}{c}\mathrm{A} \\
\text { Lóg }\end{array}$ & $\begin{array}{c}\mathrm{B} \\
\mathrm{Sec}\end{array}$ & $\begin{array}{c}\mathrm{C} \\
\text { Soc }\end{array}$ & $\begin{array}{c}\mathrm{D} \\
\text { Ima }\end{array}$ \\
\hline 1. Liderar o integrar equipos de trabajo & & & $\mathrm{X}$ & \\
\hline $\begin{array}{l}\text { 2. para el desarrollo de soluciones de } \\
\text { software. }\end{array}$ & $\mathrm{X}$ & & & \\
\hline 3. Planeación, & & $\mathrm{X}$ & & \\
\hline 4. análisis, & $\mathrm{X}$ & & & \\
\hline 5. diseño, & $\mathrm{X}$ & & & \\
\hline 6. implementación & & $\mathrm{X}$ & & \\
\hline $\begin{array}{l}\text { 7. e implantación de soluciones de redes } \\
\text { en organizaciones. }\end{array}$ & & $\mathrm{X}$ & & \\
\hline $\begin{array}{l}\text { 8. Asesoría en el uso y planeación de } \\
\text { inversión en tecnología; }\end{array}$ & & & $\mathrm{X}$ & \\
\hline 9. planeación, & & & & $\mathrm{X}$ \\
\hline 10.dirección, & $\mathrm{X}$ & & & \\
\hline 11.ejecución & & $\mathrm{X}$ & $\mathrm{X}$ & \\
\hline $\begin{array}{c}\text { 12.y evaluación de proyectos de tecnología } \\
\text { informática }\end{array}$ & $\mathrm{X}$ & $\mathrm{X}$ & & \\
\hline
\end{tabular}

\section{UNIVERSIDAD Y}

Programa Ingeniería de Sistemas

\section{PERFIL PROFESIONAL}

El ingeniero de sistemas y computación de la Universidad Y es un profesional ético, responsable, crítico y autónomo; con facilidad para integrar equipos interdisciplinarios y con capacidad para plantear, desarrollar, implantar, gestionar y evaluar alternativas de solución a problemas, necesidades y oportunidades de la sociedad y organizaciones de distintos sectores de la economía relacionadas con procesos y manejo de la información mediante la aplicación de sus conocimientos en ciencias básicas y tecnologías de la información y las comunicaciones.

\section{PERFIL OCUPACIONAL}

- Ingeniería de software. Liderar o integrar equipos de trabajo para el desarrollo de soluciones de software.

- Infraestructura de tecnología informática. Planeación, análisis, diseño, implementación e implantación de soluciones de redes en organizaciones.

- Gestión y organizaciones. Asesoría en el uso y planeación de inversión en tecnología; planeación, dirección, ejecución y evaluación de proyectos de tecnología informática.

Se desglosarán estas definiciones para poder realizar un análisis más claro en relación con los parámetros del modelo 4Q tal como se presenta en la Tabla IIA.

TABLA IIC

\begin{tabular}{|c|c|c|c|c|c|}
\hline Perfil & Ítem & $\begin{array}{c}\text { Cuad } \\
\text { A } \\
\text { Lóg }\end{array}$ & $\begin{array}{c}\text { Cuad } \\
\text { B } \\
\text { Sec }\end{array}$ & $\begin{array}{c}\text { Cuad } \\
\text { C } \\
\text { Soc }\end{array}$ & $\begin{array}{c}\text { Cuad } \\
\text { D } \\
\text { Imag }\end{array}$ \\
\hline \multirow{15}{*}{$\begin{array}{l}\mathrm{P} \\
\mathrm{R} \\
\mathrm{O} \\
\mathrm{F} \\
\mathrm{E} \\
\mathrm{S} \\
\mathrm{I} \\
\mathrm{O} \\
\mathrm{N} \\
\mathrm{A} \\
\mathrm{L}\end{array}$} & 1 & $X$ & & & \\
\hline & 2 & & & $X$ & \\
\hline & 3 & & $\mathrm{X}$ & & \\
\hline & 4 & $\mathrm{X}$ & & & \\
\hline & 5 & & & $\mathrm{X}$ & \\
\hline & 6 & & & & $\mathrm{X}$ \\
\hline & 7 & & $\mathrm{X}$ & & \\
\hline & 8 & $\mathrm{X}$ & & & \\
\hline & 9 & & & $\mathrm{X}$ & \\
\hline & 10 & $\mathrm{X}$ & & & \\
\hline & 11 & & & $\mathrm{X}$ & \\
\hline & 12 & $\mathrm{X}$ & & & \\
\hline & 13 & & $\mathrm{X}$ & & \\
\hline & 14 & $\mathrm{X}$ & & & \\
\hline & 15 & & $X$ & & \\
\hline & 1 & & & $X$ & \\
\hline \multirow{3}{*}{$\begin{array}{l}\mathrm{O} \\
\mathrm{C} \\
\mathrm{U}\end{array}$} & 2 & $\mathrm{X}$ & & & \\
\hline & 3 & & $\mathrm{X}$ & & \\
\hline & 4 & $\mathrm{X}$ & & & \\
\hline \multirow{2}{*}{ P } & 5 & $\mathrm{X}$ & & & \\
\hline & 6 & & $\mathrm{X}$ & & \\
\hline \multirow{2}{*}{ I } & 7 & & $\mathrm{X}$ & & \\
\hline & 8 & & & $\mathrm{X}$ & \\
\hline \multirow{2}{*}{$\begin{array}{l}\mathrm{N} \\
\mathrm{A}\end{array}$} & 9 & & & & $\mathrm{X}$ \\
\hline & 10 & $\mathrm{X}$ & & & \\
\hline \multirow[t]{2}{*}{$\mathrm{L}$} & 11 & & $\mathrm{X}$ & $\mathrm{X}$ & \\
\hline & 12 & $\mathrm{X}$ & $\mathrm{X}$ & & \\
\hline \multicolumn{2}{|c|}{ TO T A LES } & 11 & 9 & 7 & 2 \\
\hline \multicolumn{2}{|c|}{ PORCENTAJES } & $38 \%$ & $31 \%$ & $24 \%$ & $7 \%$ \\
\hline
\end{tabular}

Debe admitirse que la relación posible que se establece entre los componentes conceptuales del perfil profesional es completamente discutible. De allí el por qué se hace tan relevante, en estos temas, la participación activa del comité curricular respectivo. De las discusiones que se hagan, de las discrepancias y coincidencias que surjan y de las conclusiones 
e inferencias a las cuales se llegue, dependerá completamente la coherencia y articulación entre los perfiles (profesional y ocupacional), el plan de estudios y los mecanismos y criterios de admisión para los que aspiran a ingresar al programa que se ha tomado como referente de análisis. La tabla IIB presenta el análisis similar pero aplicado al perfil ocupacional tal como lo tiene establecido la Universidad Y.

La tabla IIC presenta los resultados resumidos del análisis realizado a los perfiles profesional y ocupacional (laboral) que se han tomado como ejemplo.

Estos porcentajes revelan la proporcionalidad de los componentes en los perfiles profesional y ocupacional a la luz del modelo 4Q que, de la misma forma, establecen la proporción que debe contemplarse en el plan de estudios y la relevancia de la mirada lógica, secuencial, social e imaginativa en los elementos de juicio que deben involucrarse al momento de diseñar los mecanismos y criterios de acceso al programa Ingeniería de Sistemas en esta Universidad Y.

\section{V.DISCUSIÓN}

Cualquier metodología impregnada de valoraciones que involucran lo cualitativo, también está preñada de discusiones, discrepancias y coincidencias. La metodología que se plantea en el presente artículo no es la excepción y, para bien, son esas discusiones alrededor de ella las que, a la postre, permitirán que se enriquezca, se nutra y se estructure como una metodología alimentada de la experiencia de otros, de otras visiones y de otras formas de mirar la formación universitaria de pregrado.

Las tablas IIIA y IIIB presentan el resumen de la relación posible entre el modelo 4Q y cada uno de los perfiles que se quieren alcanzar. Si lo que se persigue en esta investigación es encontrar un mecanismo científico que brinde algún tipo de garantía para que el plan de estudios y todo el proceso de formación se articule con los perfiles profesional y ocupaciones y que, además, esto se vea reflejado en la selección de los aspirantes a la carrera para bienestar y salud financiera de la universidad, sea cual fuere, entonces podría decirse que este modelo valdría la pena, por lo menos, analizarlo en los comités curriculares pues permite que se extienda una línea conductora entre los extremos de un procesos de formación profesional. De un lado están los aspirantes, de otro lado están los egresados que, finalmente, son producto de su paso del plan de estudios y son quienes van a esgrimir su perfil profesional y ocupacional como la impronta que los identifique ante la sociedad como hijos ingenieros de una determinada universidad.

La información cuantitativa que presentan las tablas IIIA y IIIB evidencian, por lo menos, la posibilidad que se tiene de articular el perfil profesional y ocupacional que se ofrece en un programa de ingeniería con un camino científico y lógico que se evidencia en su plan de estudios y en el respaldo que le brinde un modelo validado y aceptado como el modelo 4Q de preferencias de pensamiento.

Vale la pena tener en cuenta que existen otros modelos de interpretación y que cada uno podría brindar elementos de juicio, válidos y coherentes, en este tipo de metodologías sin embargo dada su gran aceptación internacional y su validación en diferentes tipos de proceso de perfilamiento que incluyen no solo lo personal sino también lo institucional, lo grupal y lo empresarial, se ha escogido este modelo como una manera de aproximarse, desde la ciencia, hacia los objetivos que se persiguen en cuanto al proceso de formación universitaria en mención.

TABLA IIIA RESUMEN DE RESULTADOS UNIVERSIDAD X - PERFILES

\begin{tabular}{|c|c|c|c|c|}
\hline Perfil & $\begin{array}{l}\text { Cuad A } \\
\text { Lóg }\end{array}$ & $\begin{array}{c}\text { Cuad B } \\
\text { Sec }\end{array}$ & $\begin{array}{c}\text { Cuad C } \\
\text { Soc }\end{array}$ & $\begin{array}{c}\text { Cuad D } \\
\text { Imag }\end{array}$ \\
\hline \multirow{2}{*}{$\begin{array}{l}\text { PERFIL PROF } \\
\text { TOTAL }\end{array}$} & 2 & 2 & 2 & 1 \\
\hline & $29 \%$ & $29 \%$ & $29 \%$ & $13 \%$ \\
\hline \multirow{2}{*}{$\begin{array}{l}\text { PERFIL OCUP } \\
\text { TOTAL }\end{array}$} & 2 & 1 & 2 & 1 \\
\hline & $34 \%$ & $16 \%$ & $34 \%$ & $16 \%$ \\
\hline TOT A L E S & 4 & 3 & 4 & 2 \\
\hline PORCENTAJES & $33 \%$ & $25 \%$ & $25 \%$ & $16 \%$ \\
\hline
\end{tabular}

TABLA IIIB

RESUMEN DE RESULTADOS UNIVERSIDA Y - PERFILES

\begin{tabular}{|c|c|c|c|c|}
\hline \multirow{2}{*}{ Perfil } & $\begin{array}{c}\text { Cuad A } \\
\text { Lóg }\end{array}$ & $\begin{array}{c}\text { Cuad B } \\
\text { Sec }\end{array}$ & $\begin{array}{c}\text { Cuad C } \\
\text { Soc }\end{array}$ & $\begin{array}{c}\text { Cuad D } \\
\text { Imag }\end{array}$ \\
\hline $\begin{array}{c}\text { PERFIL PROF } \\
\text { TOTAL }\end{array}$ & 6 & 4 & 4 & 1 \\
\cline { 2 - 5 } & $40 \%$ & $27 \%$ & $27 \%$ & $6 \%$ \\
\hline $\begin{array}{c}\text { PERFIL OCUP } \\
\text { TOTAL }\end{array}$ & 5 & 5 & 3 & 1 \\
\hline T O T A L E S & $38 \%$ & $38 \%$ & $22 \%$ & $2 \%$ \\
\hline PORCENTAJES & $38 \%$ & $31 \%$ & $24 \%$ & $7 \%$ \\
\hline
\end{tabular}

De la misma forma, el modelo $4 \mathrm{Q}$ contiene cuatro componentes que, de una u otra forma, tienen relación directa con los procesos de formación universitario como el cuadrante lógico, el cuadrante secuencial, el cuadrante social y el cuadrante imaginativo y que abren la puerta a las dimensiones lógico-matemático, secuencial, humanístico y artístico que se requieren en tiempos modernos para que se pueda recibir una formación en ingeniería acorde con las necesidades de formación en habilidades y competencias que la sociedad actual exige. Tanto en la Universidad $\mathrm{X}$ como en la Universidad Y (nominadas de esta forma para respetar su identificación formal) se puede observar que el componente que más se exige, según sus respectivos perfiles profesional y ocupacional, es el componente lógico lo cual cobra un sentido muy directo con el significado del conocimiento que subyace a la Ingeniería de Sistemas y Computación.

En segundo lugar, en la Universidad $X$ tiene alta relevancia tanto el perfil secuencial como el perfil social, ambos en igual proporción $(25 \%)$ sin embargo en la Universidad $\mathrm{Y}$ tiene mucha más relevancia el perfil secuencial por razones propias de la definición autónoma que tienen los comités curriculares y las universidades al respecto de sus propios lineamientos académicos en relación con la estructuración de sus programas. En ambas universidades, el perfil menos requerido es el imaginativo que, en posteriores discusiones, valdría la pena revisar detenidamente puesto que en un programa como Ingeniería de Sistemas contar con un plan de estudios que promueva, capitalice y aproveche la imaginación de los estudiantes podría ser el primer paso para que los otros perfiles se fortalezcan, se articulen y les brinden un soporte profesional sólido a los otros tres perfiles, pero eso será tema de otra discusión. 


\section{CONCLUSIONES}

En relación con el objetivo del presenta artículo el cual era proponer una metodología rigurosa, científica y coherente que permita encontrar mayor efectividad a todos los esfuerzos de formación y curriculares que se hacen desde la administración académico administrativa de un programa como Ingeniería de Sistemas a partir de la adopción de un modelo científico que posibilite la articulación entre el perfil profesional y ocupacional con el plan de estudios, puede decirse que la metodología propuesta cumple con lo requerido dado que, de una parte, adopta un modelo científico aceptado internacionalmente tanto a nivel individual como a nivel grupal y que se conoce como el modelo $4 \mathrm{Q}$ de preferencias de pensamiento.

De otra parte, sirve como base para establecer proporciones porcentuales de los perfiles en mención a partir del perfil lógico, secuencial, social e imaginativo, brindando herramientas conceptuales para que estas proporciones porcentuales se reflejen en el plan de estudios y establece criterios claros y definidos para que las pruebas de admisión que se adopten para ingresar al programa de Ingeniería estén en sintonía con lo que se pretende lograr en el plan de estudios y que se define en los perfiles profesional y ocupacional.

La puerta queda abierta para las discusiones a que haya lugar pues la simple selección del modelo $4 \mathrm{Q}$ de preferencias de pensamiento obedece a un conjunto de criterios que pueden analizarse en relación con otros modelos que también pueden enriquecer los procesos de estructuración de un plan de estudios de un programa de Ingeniería y de definición de los perfiles profesional y ocupacional, así como los criterios de admisión de los aspirantes al programa en mención. Se concluye que, a partir de un modelo científico validado, probado y aceptado, es posible lograr lo que se ha planteado como objetivo en la presente investigación que es lo que ha inspirado el artículo que aquí se presenta.

\section{REFERENCIAS}

[1] O. Trejos Buriticá, Tesis Doctoral "Aprendizaje en Ingeniería: Un problema de Comunicación", Pereira: Doctorado en Ciencias de la Educación CADE UTP, 2012.

[2] S. Phillips y K. Kisner, Accreditation on the Edge: Challenging Quality Assurance in Higher Education, Baltymore (Maryland): Johns Hopkins University Press, 2018.

[3] M. Lumsdaine y E. Lumsdaine, Creative Solving Problem, Miami: McGraw Hill Companies, 2005.

[4] R. Falcao, Improving learning outcomes assessment in Engineering Education, Lovaina (Bélgica): Universidad Nova de Lisboa - ENAEE Members Forum 2017, Nov 2017.

[5] J. De Zubiría Samper, «El papel de la investigación en la consolidación de las innovaciones,» Revista Educación y Ciudad (IDEP), $\mathrm{n}^{\circ} 32$, pp. Págs 15-21, 15 Junio 2017.

[6] W. Herrmann, The whole brain bussiness book, New York: McGraw Hill, 2015.

[7] Programa Regional de Formación Ocupacional, Metodología para la elaboración del diseño curricular, San José de Costa Rica: Coordinación Educativa y Cultural Centroamericana, sep 2009.

[8] A. d. M. Lalor, Ensuring High-Quality Curriculum: How to Design, Revise, or Adopt Curriculum Aligned to Student Success, Ciudad de México: ASCD, 2016.

[9] A. Agrawall, The 4Q framework, New York: Independient Publishing, 2018.

[10] S. Rosini y R. Tagliabue, El pensamiento científico, Buenos Aires :
Biblos Editorial, 2010.

[11] E. Lumsdaine y M. Lumsdaine , Creative Solving Problem, 3a Ed. ed., New York: McGraw Hill, 1994, p. 491.

[12] F. Diaz Barriga y G. Hernandez Rojas, Estrategias docentes para un aprendizaje significativo, México: McGraw Hill, 2002.

[13] M. C. Tumino y J. M. Bournissem , Competencias profesionales y el paradigma del conectivismo: Hacia la formación profesional, Barcelona: Editorial Académica Española, 2016.

[14] J. E. Rivera, Cómo diseñar planes de estudio, Bogotá: Editorial Magisterio, 2010.

[15] J. De Zubiría Samper, Las competencias argumentativas: la visión desde la educación, Bogotá: Editorial Magisterio, 2013.

[16] W. Herrmann, «Herrmann Brain Dominante Instrument,» HBDI Inc, jun 2019. [En línea]. Available: www.hbdi.com. [Último acceso: jun 2019].

[17] P. Benziger, «Pruebas Benziger,» Benziger Corp, Jun 2019. [En línea]. Available: www.benziger-la.com. [Último acceso: Jun 2019].

Omar Iván Trejos Buriticá. Ingeniero de Sistemas. Especialista en Instrumentación Física. MSc en Comunicación Educativa. PhD en Ciencias de la Educación. Docente de Planta, Facultad de Ingenierías, Ingeniería de Sistemas y Computación. Área de Programación.omartrejos@utp.edu.co

OrCid: https://orcid.org/0000-0002-3751-6014.

Luis Eduardo Muñoz Guerrero. Ingeniero de Sistemas. MSc en Ingeniería de Sistemas. PhD (E) en Ciencias de la Educación. Docente de Planta, Facultad de Ingenierías, Ingeniería de Sistemas y Computación. Área de Programación.lemunozg@utp.edu.co

OrCid: http://orcid.org/0000-0002-9414-6187.

Guillermo Roberto Solarte Martínez. Ingeniero de Sistemas. MSc en Administración Económica y Financiera. $\mathrm{PhD}$ en Ingeniería Informática. Docente de Tiempo Completo, Facultad de Ingenierías, Ingeniería de Sistemas y Computación, Universidad Tecnológica de Pereira. Area de Programación. solarte@utp.edu.co

OrCid: https://orcid.org/0000-0003-2538-8286. 